\title{
THE HUAIHE BASIN WATER RESOURCE AND WATER QUALITY MANAGEMENT PLATFORM IMPLEMENTED WITH A SPATIO-TEMPORAL DATA MODEL
}

\author{
Yuanming LIU ${ }^{\text {a }}$, Wanchang ZHANG ${ }^{\text {a, b, * }}$, Changan YAN ${ }^{\text {a }}$ \\ ${ }^{a}$ State Key Laboratory of Pollution Control \& Resource Reuse, School of Environment, Nanjing University, Nanjing, \\ 210093, China.ymliu@live.com \\ ${ }^{\mathrm{b}}$ Centre for Earth Observation \& Digital Earth, Chinese Academy of Sciences, Beijing, 100094, China.
}

KEY WORDS: Watershed management, WebGIS, Object-oriented spatio-temporal data model, Environmental assessment, Decision-making support, Online hydrological simulation

\begin{abstract}
:
Presently, planning and assessment in maintenance, renewal and decision-making for watershed hydrology, water resource management and water quality assessment are evolving toward complex, spatially explicit regional environmental assessments. These problems have to be addressed with object-oriented spatio-temporal data models that can restore, manage, query and visualize various historic and updated basic information concerning with watershed hydrology, water resource management and water quality as well as compute and evaluate the watershed environmental conditions so as to provide online forecasting to police-makers and relevant authorities for supporting decision-making. The extensive data requirements and the difficult task of building input parameter files, however, has long been an obstacle to use of such complex models timely and effectively by resource managers. Success depends on an integrated approach that brings together scientific, education and training advances made across many individual disciplines and modified to fit the needs of the individuals and groups who must write, implement, evaluate, and adjust their watershed management plans. The centre for Hydro-science Research, Nanjing University, in cooperation with the relevant watershed management authorities, has developed a WebGIS management platform to facilitate this complex process. Improve the management of watersheds over the Huaihe basin through the development, promotion and use of a web-based, user-friendly, geospatial watershed management data and decision support system (WMDDSS) involved many difficulties for the development of this complicated System. In terms of the spatial and temporal characteristics of historic and currently available information on meteorological, hydrological, geographical, environmental and other relevant disciplines, we designed an object-oriented spatiotemporal data model that combines spatial, attribute and temporal information to implement the management system. Using this system, we can update, query and analyze environmental information as well as manage historical data, and a visualization tool was provided to help the user interpret results so as to provide scientific support for decision-making. The utility of the system has been demonstrated its values by being used in watershed management and environmental assessments.
\end{abstract}

\section{INTRODUCTION}

In recent years, environment deterioration associated with rapid social-economic development in China has acquired more and more attention. Research and practice of environmental protection have proved that to effectively curb environmental pollution the engineering measures should be combined with the managing methods closely. One important goal of the basin management information system (MIS) is to systematically reveal the natural ecological and environmental basic features of basin so that people can accurately hold the key problems to control pollution (Simonovic 1999) (Ludwig et al. 2003). Academically, MIS is used to refer to the group of information management methods tied to the automation or support of human decision making (O'Brien and Marakas 2005). With the rapid development of Internet and maturity of database technology, MIS extends from Client/Server $(\mathrm{C} / \mathrm{S})$ architecture to Browser/Server (B/S) architecture (Isakowitz et al. 1998).

In the real world, the basin MIS is closely linked to our society. The information of water quality should be released to the public timely through Internet for agricultural irrigation and industrial production. To make accurate decision, relevant authorities need to wholly review reports based on environmental data warehouse. The historic monitoring data drives environmental simulation whose forecasting result directly supports the basin planning and management. Along with the spread of Internet, a growing number of people pay attention to the environment protection and sustainable development (Guo et al. 2001). Therefore, launching the research of basin MIS is an urgent demand to guarantee sustainable development of society and economy within basin. As a typical application of MIS, Digital Basin and Digital River have gone through years of construction (Jamieson and Fedra 1996) (Rousseau et al. 2000) (Rousseau et al. 2000). Famous MIS in it are Mississippi basin MIS, Loire Valley MIS, Rhine Valley MIS and Murray-Darling basin MIS (Locke 2004) (Cecarelli et al. 2001).

These previous research works can be characterized according to the following criteria:

- The spatio-temporal data model is taken into account, but there is a serious weakness of existing environmental models that each of them deals with few common characteristics found across a number of specific applications, which means that the research aims at the special environment problems of basin but a object-oriented spatio-temporal data model to universal phenomena of basin environment is lacked. Thus the applicability of model to different cases fails on spatio-temporal

\footnotetext{
${ }^{*}$ Corresponding author. zhangwc@nju.edu.cn
} 
behaviors not anticipated by the application used for the initial model development (Pelekis et al. 2004).

- The construction of resource layer has not yet rise to the level of spatio-temporal data warehouse but actually spatiotemporal database of basin, which results in poor reliability for supporting decision making.

- There are complex offline hydrological simulation based on GIS and simple online simulation based on WebGIS, such as SWAT, BASINS and AGWA (Duda et al. 2003). Based on spatial technology, SWAT is used to predict the impact of land management practices on water, sediment and agricultural chemicals yields in large complex watersheds with varying soils, land use and management conditions over long periods of time (Arnold and Fohrer 2005). As a multipurpose hydrological analysis system, AGWA is widely used by watershed, natural resource, and land use managers and scientists in performing basin-scale studies (Miller et al. 2007).

- Only simple business logic modules are realized, and the needs of basin management especially decision-making support have not yet deeply analyzed (Winchell et al. 2008).

- How to closely integrate the library of environmental models with environmental data warehouse in online analytical processing (OLAP) requires further investigation, such as roll up and drill down in line with the requirements of environmental simulation (Inmon 2005).

- For beginners most models with non-friendly interfaces cause difficulty to operate smoothly. Meanwhile, because hydrological models take traditional $\mathrm{C} / \mathrm{S}$ architecture, it is difficult to update features timely and conveniently.

To the base of our knowledge, online simulation is a brand-new pattern so that there is a long way to go before running the web business on basin MIS. Based on this categorization, we can characterize our basin MIS as follows:

- According to the features of water environment, an objectoriented spatio-temporal data model is set up. Various computing models are optimized and then encapsulated into an environmental library. Furthermore, the spatio-temporal warehouse we propose is closely integrated with the library of environmental models by public interfaces.

- The library of environmental models couples with WebGIS. Spatio-temporal query technique, overlay analysis method and real-time map presentation play active role throughout all stages of hydrological simulation. Here a WebGIS provides the framework within which spatially distributed data is collected and used to pre-process model input data and evaluate model results (Miller et al. 2007). We insist that online simulation based on spatial technology should be taken enough respect so that the whole calculation process can be expressed clearly in spatio-temporal variation of environmental data.

- The basin MIS forms a linkage mechanism among data warehouse, library of environmental models and decisionmaking support.

- Because the basin MIS takes popular B/S architecture, all kinds of users can conveniently execute corresponding operations via the user-friendly portal. The basin MIS has the potential of reaching a much wider audience (Isakowitz et al. 1998). And it is not like other experimental type of research but has been successfully applied to Huaihe Basin, China.

\section{FRAMEWORK OF BASIN MIS}

\subsection{Resource Layer}

The data from various sources is distributed, heterogeneous, multi-dimensional and multi-layered, which is difficult to be used directly by environmental simulation or decision-making process. However, data warehouse can manage the subjectoriented, integrated, non-volatile and time-variant collection of data to significantly improve the efficiency of raw data. Meanwhile, the data warehouse has been a key part of decision support system (DSS) (Inmon 2005). The experiment indicates that a higher level of decision-making can be reached well with the support of the data warehouse (Park 2006). Therefore, the environmental data warehouse is the basis of information service, online hydrological simulation and spatial decision support of basin MIS.

\subsubsection{Object-oriented spatio-temporal data model}

It is recognized that the morphology, topology and attributes of a spatio-temporal object may or may not change over time, allowing for eight different scenarios (Pelekis et al. 2004). The environmental data of basin such as rainfall, runoff and pollutant migration has obvious time attribute, and its transformation is a continuous differentiable process. However, most database management system (DBMS) collects discrete data to record continuous change. Likewise, environmental simulation models such as rainfall-runoff models often use discrete analog computation to interpret rainfall-runoff process and pollutant diffusion process. If the spatial location, text attribute, spatial relation and time of research object are concurrently considered, the data processing would be very difficult. For this problem, there is a kind of time expression pattern where data is processed by fixing a defined item, limiting a controlled item and measuring a variable item as shown in Table 1 (Sinton 1978).

\begin{tabular}{|l|l|l|l|}
\hline Object & Defined item & Controlled item & Variable item \\
\hline Pollutant & Location & Time & Attribute (content of pollutant) \\
Flood & Location & Time & Attribute (water level) \\
Vegetation & Time & Attribute (type) & Location \\
Land & Time & Location & Attribute (type) \\
\hline
\end{tabular}

Table 1. Traditional time expression pattern

However, the traditional time expression pattern above is difficult to describe the moving objects and its defined item, controlled item and variable item. Therefore, a object-oriented spatio-temporal data model is needed to describe the features of environment entities, which has ability to constantly track certain entity in space and time, analyze its history and dynamically query its state, such as dynamic management, pollutant migration analysis and disaster forecast. The common data model is also a tendency of environment MIS development.

Besides the flaw above, there is another problem about data format that should be paid attention too. Actually, the abstract data model masks the internal difference of data formats. Take vector data model composed of discrete features for example, there are various data formats from ArcGIS, MapInfo and so on, and so do raster data and relational data (Zeiler 1999). In addition, the spatial location information (spatial data, represented by vector data model and raster data model) and attribute information (relational data, represented by relational data model) are separately stored. Part of these reasons is to be convenient for storing and retrieving data, but that is not appropriate for basin MIS to couple database with the library of environmental models based on WebGIS.

To accurately describe the moving objects and integrate various data features into one data model, we propose an objectoriented spatio-temporal data model where the features of the environmental entities are also emphasized, as follows: 


\section{$<$ OBJECT: \{Identity (ID), Spatial (s), Temporal $\left(\mathrm{T}_{\mathrm{t}}, \mathrm{T}_{\mathrm{v}}\right)$,} Actions $\}>$

Every environmental object is composed of identity, spatial information, attribute collection and actions. Identity (ID), a unique code to identify an object, remains constant during its lifetime. Spatial (s) describes the spatial information varying with time. The attribute collection encapsulates thematic attribute and spatio-temporal attribute. The thematic attribute either text or image describes the characters and features of object. The temporal attribute records the change of object's tense, and it has a valid time Temporal $\left(\mathrm{T}_{\mathrm{v}}\right)$ when the object was created, varied and dead, which means that the valid time (realworld time) $T_{v}$ describes the time that an event actually happened in the real world; and the transaction time (registration time) Temporal $\left(\mathrm{T}_{\mathrm{t}}\right)$ indicates the time an event is actually recorded in the database. $T_{t}$ and $T_{v}$ are orthogonal to each other. Actions contain various operations on an object. Here we take pseudo code of monitoring station entities for example as follows:

Class MonitoringStation

\{

// Name of a monitoring station

StationName;

// Unique identity code of a station.

// The monitoring category can be identify from the code. StationID;

// Unique identity code of a layer containing the station.

LayerID;

// Geographic coordinates of a station.

Location $(\mathrm{x}, \mathrm{y})$;

// Time to create a station in the shape of point.

SpatialCreateTime;

// Array that records the time to modify a station as a point. SpatialModifyTimeArray;

// Array that records the time to delete a station as a point. SpatialDeleteTime;

// Arrys that records the attribute of a station.

AttributeArray;

// Time to originally add the attribute to a station.

AttributeCreateTime;

// Time to modify the attribute to a station.

AttributeModifyTimeArray;

// Time to create a station as a point will be returned.

SpatialCreate(x, y, LayerID);

// Time to modify a station as a point will be returned.

SpatialModify(x, y, LayerID);

// Time to delete a station as a point will be returned.

SpatialDelete(x, y, LayerID);

// Time to add the attribute to a station will be returned.

AttributeCreate(AttributeArray);

// Time to modify the attribute to a station will be returned. AttributeModify(AttributeArray);

// Query based on attribute.

SearchStation(StationID);

// Query based on space.

SearchStation(x, y);

// Query based on space and time.

SearchStation(x, y, SpatialCreateTime);

\}

\subsubsection{Environmental data warehouse for decision-making} support

Data from various sources is distributed, heterogeneous, multidimensional and multi-layered, which is difficult to be used directly by hydrological simulation or decision-making process before being stored into database without cleaning it up (Sheth and Larson 1990). According to our statistics, the data collected contains: 1:4,000,000 basic geographic and hydrological data, social economy and environmental protection data, hydrological monitoring data, water quality monitoring data, water environment model information and basin knowledge information database, and so on. The thematic data is extracted, transformed and then integrated into reference dataset of basin, monitoring dataset, hydrological network dataset, environmental feature dataset, raster feature dataset and relational dataset respectively.

The relevant criterion for handling data contain: coding standard, spatial references coordinate system standard, symbol standard, data format specification, data quality standard, metadata standard and technical standard of environmental database. After seriously evaluating data quality, all spatiotemporal data is gradually stored in our environmental database at server with the support of Geo-database of ArcGIS, as shown in Figure 1 (Zeiler 1999). This object-oriented spatio-temporal database can assist system administrator in managing basic data of whole basin, and can also be called remotely by interfaces via local metadata specification.

(a)

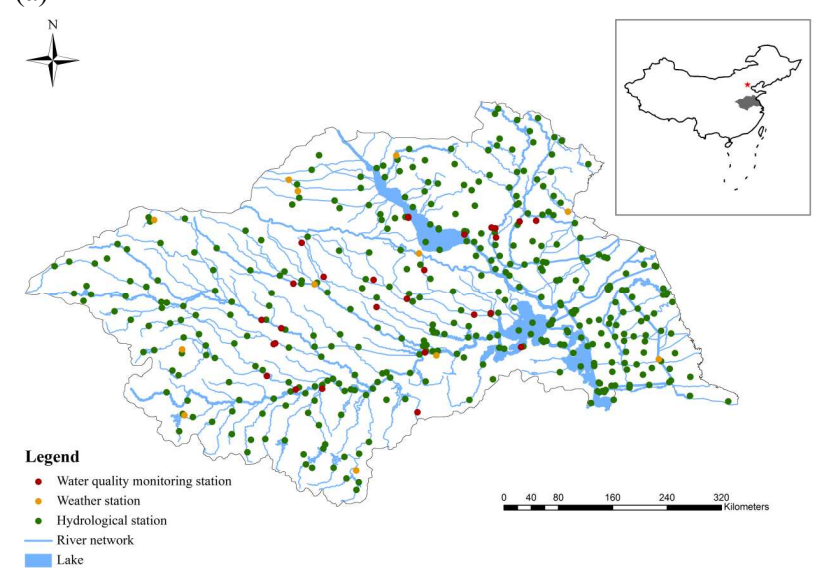

(b)

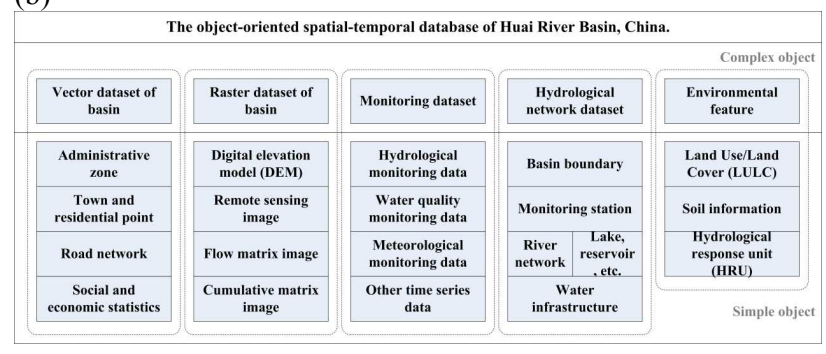

Figure 1. (a). Map of Huaihe Basin, China. (b). The objectoriented spatio-temporal database

According to the object-oriented spatio-temporal database, the data warehouse is set up to provide the decision-making support with adequate refined data. The detailed method as follows:

- Firstly, the data is extracted, transformed and loaded via the extract-transform-load process (ETL) from the object-oriented database. The ETL is an important process in database usage and especially in data warehousing that involves extracting data from outside sources, transforming data to fit operational needs and at last loading data into the container at reconciling layer before the new data is being loaded into the data market. The advantage of reconciling layer is that the common data model is established here that helps to divide the process of filling up 
data warehouse from the process of extracting and integrating data from the original database (Kimball 2006).

- The temporary data stored at reconciliation layer is integrated, consistent, accurate, newest and detailed. The data market is subject-oriented, and it should meet the special needs of the professional user groups. According to the demand analysis, there are three data markets namely water environment, geographical environment and reference of basin, deriving from the reconciling layer. The data warehouse can provide reorganized data to the analysis process such as OLAP, data mining, hypothesis analysis and so on. However, the environmental simulation needs the involvement of database because it is driven by the raw observation data.

- The result including reports, tables and other environmental forecast is directly adopted by application of decision-making support, such as finding out the current pollution load of the basin and forecasting the changing tendency of water quality in future.

The fact and dimension table of environmental data warehouse are shown in Figure 2, which includes three subjects (water environment, geographical environment and reference of basin) and twelve dimensions (time, geography, station, statistics, hydrology, quality, basin, river, infrastructure, disaster, reservoir and meteorology).

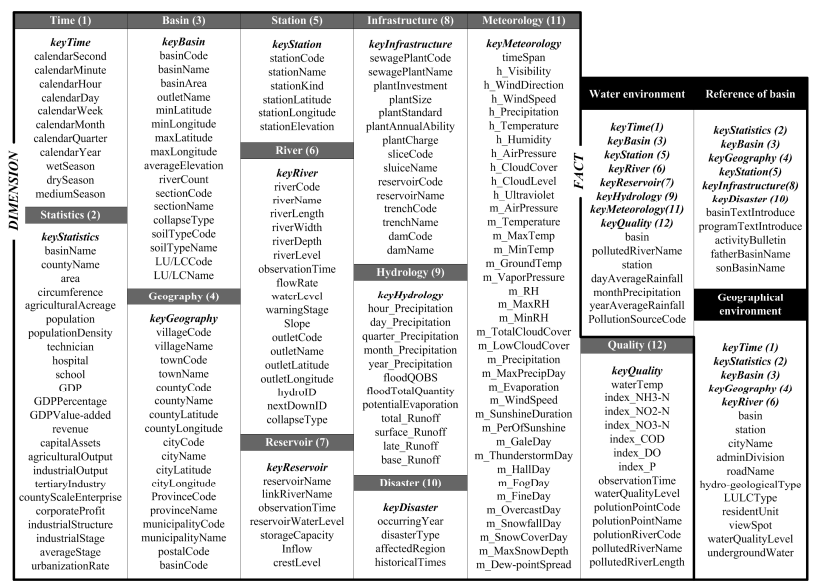

Figure 2. The fact and dimension table of data warehouse

And we take the surrogate key instead of various independent keys from corresponding tables, which provides a recommended way to deal with the concern and is used to relate the fact table to the dimension table (Kimball et al. 2011). As shown in Figure 3, the original coding system of database is not used again, and the new agent key combined reasonably is filled of the integral number without any meaning.

\subsection{Business Logic Layer}

Before planning and managing water resource of basin, managers often look forward to the support from the professional simulation that has the ability to analyze, evaluate and forecast to the environment problems. Presently, various environmental models have been well-developed, including the mathematical models on pollutant transport, the simulation on water temperature, the mathematical models on water quality, the pollution forecasting on rainfall runoff, and so on. However, the simulation in specific basin is subjected to difficulties. Firstly, there are so many environmental models with which researchers are often not familiar. If the improper models are chosen, resources and time might be vastly wasted but the precision of result is not satisfied. Secondly, the complex structure of input data generates a difficult challenge for researchers to process it in advance. Because there is no common object-oriented spatio-temporal data model upon the environmental features, the organizational optimization of different formats of data is rarely encouraged to consider. Thirdly, the obstacles to connection among different models still exist. Commonly, when different models at various stages on the same subject are used, the data sharing and the results contrast among these models are almost impossible, which leads to duplication of effort especially for data process. Lastly, for beginners most models with non-friendly interfaces also cause difficulty to operate smoothly.

The online simulation based on object-oriented database and WebGIS is in the front end of information technology, and has the characteristics of high technology. And establishing the library of environmental models provides a new method to resolve problems above. Optimal models within library are linked each other and can be reused in line with specified functions. In short, the library with clear topological relation avoids redundancy, and improves flexibility and efficiency significantly.

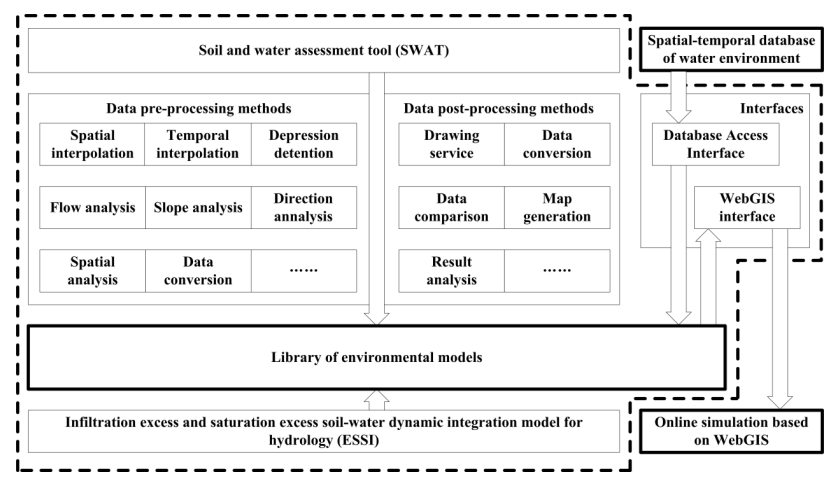

Figure 3. The technical route to establish the library of environmental models

As shown in Figure 3, the library integrates soil and water assessment tool (SWAT) and infiltration excess and saturation excess soil-water dynamic integration model for hydrology (ESSI), as well as the data pre-processing methods and the data post-processing methods. Based on the integration of infiltration excess and saturated excess runoff producing method, the results of ESSI indicate that both accuracy and efficiency are satisfactory compared with SWAT (Zhang 2006). The internal/external interfaces are developed to successfully connect individual models/modules. Now the library is directly applied to analysis and forecast of the water environment based on the rule of water quality fluctuation (modified from the open source SWAT), water pollution and self-purification model, pollutant migration and transformation equations, mathematical model of water temperature, mathematical model of water quality, parameter estimation model, non-point pollution analysis model and so on. As the hydrological model is the basis of water environment simulation, ESSI is also integrated into our library, which is being successfully applied to the hydrological simulation of Hanjiang River basin (Warm and Moist Region), Hei River basin (Cold and Arid Region), and now is being applied for patent (Zhang 2006). Meanwhile, the library provides the abundant pre-processing methods for input data such as digital elevation model data (DEM) and the postprocessing methods for output data based on spatial technology. 
Various advanced and matured techniques especially objectoriented programming (OOP) are also broadly applied to the steps of modularization design and integration analysis.

By integrating ESSI with SWAT and realizing other algorithms involved, the library can fundamentally solve the problems during the models selection, data management and connection among the models, and at last provide the managers with a powerful support for decision making.

\subsection{Presentation Layer}

At presentation layer, the business system planning method (BSP) is used to deal with data, processes, strategies, aims and organizational departments which are interconnected. Analytical part about organizational structure, business strategy and data classes is the most important part of BSP (Zachman 1982). The requirements of different users are taken into account firstly, and the architecture is set up in concept designed from top to bottom; according to the coverage of the environmental dataset, we develop the basin MIS in function from bottom to top. During the requirement analysis stage, we interview with different users to clearly understand their requirements. Especially for basin departments and managers, we train them about the knowledge of data warehouse so that the users' mind and perspective are enlighten and make sure that their dynamic requirements keep within bounds of the ability of the data that we collected. Ultimately, according to these respective requirements we divide users into five groups: administrator group, the public group, office worker staff, researcher group and manager group. In order to guarantee the security of the system, we also assign different permissions and functions for corresponding groups as shown in table 2.

\begin{tabular}{|c|c|c|c|c|c|}
\hline \multirow[b]{2}{*}{$\begin{array}{l}\text { Classification of } \\
\text { functions }\end{array}$} & \multicolumn{5}{|c|}{ Permissions of different groups } \\
\hline & $\begin{array}{l}\text { Admini } \\
\text { strator }\end{array}$ & $\begin{array}{l}\text { The } \\
\text { public }\end{array}$ & $\begin{array}{l}\text { Office } \\
\text { worker }\end{array}$ & $\begin{array}{l}\text { Researc } \\
\text { her }\end{array}$ & $\begin{array}{l}\text { Manag } \\
\text { er }\end{array}$ \\
\hline $\begin{array}{l}\text { System management } \\
\text { Information retrieval }\end{array}$ & $\sqrt{ }$ & $\sqrt{ }$ & $\gamma$ & $\gamma$ & 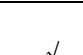 \\
\hline $\begin{array}{l}\text { Data \& function } \\
\text { update }\end{array}$ & & & $\checkmark$ & & \\
\hline Online office & & & $\checkmark$ & & \\
\hline Map service & & $\checkmark$ & $\sqrt{ }$ & $\sqrt{ }$ & $\checkmark$ \\
\hline Metadata service & & & & $\sqrt{ }$ & \\
\hline Online simulation & & & & $\sqrt{ }$ & $\sqrt{ }$ \\
\hline $\begin{array}{l}\text { Water environment } \\
\text { forecast }\end{array}$ & & & & & $\sqrt{ }$ \\
\hline Emergency response & & & & & $\sqrt{ }$ \\
\hline
\end{tabular}

Table 2. Permissions and functions of different groups

- Administrator group. The web administrator group owns the super administration authority of basin MIS. With the support of .NET frame technology, administrator can manage other users' permissions, review the data and functions updated by office worker group and distribute information via portal.

- The public group. The basin MIS provides the public group with basic information service and map service to satisfy its interest, such as information retrieval, statistical analysis, especially geo-data presentation based on WebGIS and spatiotemporal query of basin environment.

- Office worker group. The duty of office worker group is to maintain data and functions of the basin MIS regularly. They can also conveniently perform standard office procedures including scheduling appointments and processing mail on browsers now.

- Researcher group. Researchers can search the data list and then apply for the operational data from environmental database via metadata service. The metadata service is necessary when the underlying data is distributed, heterogeneous. For example, the data of our database is stored at five different nodes that belong to corresponding water management agencies. In addition, the environmental simulation is being developed and researchers can conveniently operate it without more assistance via portal. For more information, please visit http://202.119.43.91:8081/m_ESSI.aspx.

- Manager group. The result of simulation about the water environment forecast for the future is offered, which provides managers with a powerful support for decision making. We also develop other functions, such as emergency response to water pollution base on butter analysis technology.

\section{DISCUSSIONS}

The multi-layer web structure based on $\mathrm{B} / \mathrm{S}$ architecture grows up gradually, whose best advantage is that operations can be executed conveniently via browsers and the features are only updated at server. In this paper, we present a systematic procedure of database \& data warehouse - model library decision making linkage of basin MIS; especially the common data model and the dynamic library of environmental models that are proposed to realize the new online simulation based on WebGIS (see Figure 4).

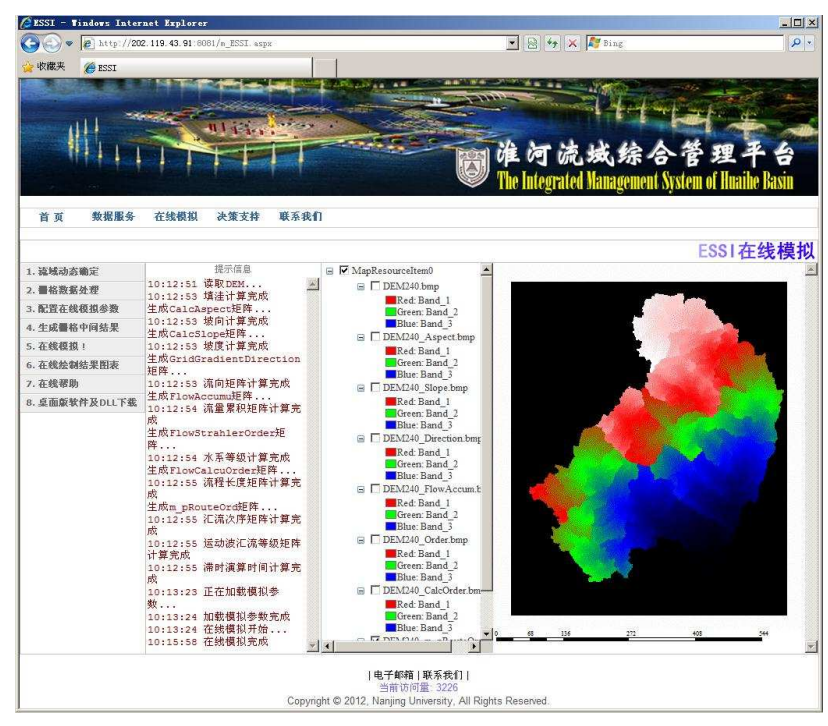

Figure 4. Online hydrological simulation of Huaihe basin MIS

We believe that the basin MIS have achieved the following points to be already feasible.

- Manage environmental data efficiently. As the complexity of environmental models and decision-making support process, how to manage the underlying data efficiently determines the success of basin MIS. The common data model accurately describes the features of dynamic environmental entities and their external relationship. Take the rainfall for example, observation data shows that rainfall intensity varies significantly across the basin. To accurately present rainfall, the objectoriented spatio-temporal data model we proposed encapsulates related attributes (location of monitoring station, observation time, total rainfall duration, observation value, etc.) and actions (rainfall spatio-temporal interpolation, rainfall forecast, pollutant concentration analysis, runoff analysis, etc.). It is able to track rainfall in space and time, search its historical records and analyze impact on other relative entities. 
- Provide the integrated business logic based on WebGIS. As a maintainable distributed GIS solution, WebGIS provides not only simple text and table, but also visual multimedia information to users; especially being excellent for spatial calculation and data management, it is very useful in hydrological simulation. Therefore, we collect the requirements by investigating users extensively, and design the integrated business logic based on spatial technology. With the support of WebGIS APIs, the simulation procedure directly fetches raster data from database to participate distributed computation and then shows the result on digital map in time, and so does the process of decision-making support.

- Save cost. By integrating ESSI with SWAT and realizing other algorithms involved, the library can solve many problems during simulation. Because the library improves flexibility and efficiency of simulation significantly, resources and time will be saved; meanwhile, the precision of result is also satisfied. Moreover, all kinds of users can conveniently execute corresponding operations via browsers since the basin MIS takes popular $\mathrm{B} / \mathrm{S}$ architecture. Because the data collection, environmental information release, online simulation, decisionmaking support and system updating are all accomplished through the Internet, the cost to maintain and implement basin MIS is lower.

\section{CONCLUSIONS AND PERSPECTIVES}

Differing from most of other MIS, the research establishes object-oriented database and spatio-temporal data warehouse at resource layer, and seamlessly integrates database with the library of environmental models based on spatial technology. As the core of basin MIS, the object-oriented spatio-temporal data model can accurately describe features of moving entities and obviously promote data access efficiency. It is proved that the common data model can save cost and time of development. To satisfy the data requirements for basin management, we explore appropriate subjects and dimensions about basin environment. The library of models contributes to computation and evaluation of the environmental conditions so as to provide organizational departments and managers of basin with decision-making support. The series of modelling methods and business logic will offer an ideal way to explore how basin MIS overcomes the weaknesses in data management and decisionmaking support. Our next work is to extend the library of environmental models to water quality. The response relationship between industrial development and water environment also will be demonstrated in decision-making support module with the aid of spatial technology.

\section{ACKNOWLEDGEMENTS}

This work is supported by the National Natural Science Foundation of China (No. 40971024) and the National Basic Research Program of China (No. 2010CB951404).

\section{REFERENCES}

ARNOLD, J. and FOHRER, N. 2005. SWAT2000: current capabilities and research opportunities in applied watershed modelling. Hydrological processes, 19(3), 563-572.

CECARELli, N., ADDISON, C. and STRICKLAND, R. M. 2001. Exploring Landscape, Documenting Culture, Constucting Memory: The Loire Valley Internet workshop. Actas de ICHIM 2001, 583-594.
DUDA, P. B., et al. 2003. BASINS 4.0 Flexible Integration of Components and Data for Watershed Assessment and TMDL Development. Proceedings of the Water Environment Federation, 2003(4), 53-68.

GUO, H., et al. 2001. A system dynamics approach for regional environmental planning and management: A study for the Lake Erhai Basin. Journal of Environmental Management, 61(1), 93-111.

INMON, W. H., 2005. Building the data warehouse. WileyIndia.

ISAKOWITZ, T., BIEBER, M. and VITALI, F. 1998. Web information systems. Communications of the $A C M$, 41(7), 78-80.

JAMIESON, D. and FEDRA, K. 1996. The 'WaterWare'decision-support system for river-basin planning. 1. Conceptual design. Journal of Hydrology, 177(3-4), 163-175.

KIMBALL, R., 2006. The data warehouse toolkit. Wiley-India.

KIMBALL, R., et al., 2011. The data warehouse lifecycle toolkit. Wiley.

LOCKE, M. A., Mississippi Delta management systems evaluation area: overview of water quality issues on a watershed scale. ed., 2004, 1-15.

LUDWIG, R., et al. 2003. Web-based modelling of energy, water and matter fluxes to support decision making in mesoscale catchments--the integrative perspective of GLOWA-Danube. Physics and Chemistry of the Earth, Parts A/B/C, 28(14-15), 621-634.

MILLER, S. N., et al. 2007. The automated geospatial watershed assessment tool. Environmental Modelling \& Software, 22(3), 365-377.

O'BRIEN, J. A. and MARAKAS, G. M., 2005. Introduction to information systems. McGraw-Hill/Irwin.

PARK, Y. T. 2006. An empirical investigation of the effects of data warehousing on decision performance. Information \& management, 43(1), 51-61.

PELEKIS, N., et al. 2004. Literature review of spatio-temporal database models. The Knowledge Engineering Review, 19(3), 235-274.

ROUSSEAU, A., et al. 2000. GIBSI-An integrated modelling system prototype for river basin management. Hydrobiologia, 422, 465-475.

SHETH, A. P. and LARSON, J. A. 1990. Federated database systems for managing distributed, heterogeneous, and autonomous databases. ACM Computing Surveys (CSUR), 22(3), 183-236.

SIMONOVIC, S. P. 1999. Decision support system for flood management in the Red River Basin. Canadian Water Resources Journal, 24(3), 203-223.

SINTON, D. 1978. The inherent structure of information as a constraint to analysis: Mapped thematic data as a case study. Harvard papers on geographic information systems, 6, 1-17.

WINCHELL, M., et al. 2008. ArcSWAT User's Guide. Temple, Tex.: Blackland Research Center, Texas Agricultural Experiment Station.

ZACHMAN, J. A. 1982. Business systems planning and business information control study: a comparison. IBM Systems Journal, 21(1), 31-53.

ZEILER, M., 1999. Modeling our world: the ESRI guide to geodatabase design. Esri Pr.

ZHANG, D., 2006. Study of Distributed Hydrological Model with the Dynamic Integration of Infiltration Excess and Saturated Excess Water Yielding Mechanism. ([D]). Nanjing University. 